\title{
An Enhanced Multi-Frequency Distorted Born Iterative Method for Ultrasound Tomography Based on Fundamental Tone and Overtones
}

\author{
Tran Quang-Huy, Hanoi Pedagogical University 2, Vietnam \\ Tuan-Khai Nguyen, University of Ulsan, South Korea \\ Vijender Kumar Solanki, CMR Institute of Technology, India \\ (iD) https://orcid.org/0000-0001-5784-1052 \\ Duc-Tan Tran, Phenikaa University, Vietnam \\ iD https://orcid.org/0000-0002-7673-388X
}

\begin{abstract}
Conventional distorted born iterative method (DBIM) using single frequency has low resolution and is prone to creating images with high-contrast subjects. The authors propose a productive frequency combination method to better result in tomographic ultrasound imaging based on the multi-frequency technique. This study uses the natural mechanism of emitting oscillators' frequencies and uses these frequencies for imaging in iterations. They use a fundamental tone (i.e., the starting frequency f0) for the first iteration in DBIM, then consecutively uses its overtones for the next ones. The digital simulation scenarios are tested with other multi-frequency approaches to prove the method's feasibility. They performed 57 different simulation scenarios on the use of multi-frequency information for the DBIM method. As a result, the proposed method is for the smallest normalization error ( $\mathrm{RRE}=$ 0.757). The proposed method's imaging time is not significantly longer than the way of using single frequency information.
\end{abstract}

\section{KEYWORDS}

Distorted Born Iterative Method, Fundamental Tone and Overtones (FTaOT), Inverse Scattering, Sound Contrast, Tomographic Ultrasound

\section{INTRODUCTION}

Medical imaging is the method of creating images of human or animal parts to collect data concerning the structure or properties of tissue, bone, or even physiological characteristics by injecting particular substances into the body (National Research Council, 2006). For the past years, medical imaging has been rapidly changing clinical diagnosis. With the advancement of media and information technology, numerous intelligent and sophisticated diagnosis and treatment methods have been presented (Feng, 2019). In 1885, Wilhelm Roentgen discovered X-ray; hence, medical imaging was born. In the past 
century, a significant change has been originated from X-ray to MRI, CT, PET, SPECT, ultrasound, etc. The efficiency of non-intrusive imaging systems has made a considerable step along with computer science.

Nowadays, there are a vast number of biological imaging methods, as mentioned above. However, in our study, we only focus on ultrasound because it is one of the most popular and often considered a golden standard in essential diagnoses such as obstetric or cardiac issues.

The use of sonar in 1910 led to the popularity of imaging techniques using sound waves later. One of the most popular sonar-based imaging methods is B-mode (Schueler et al., 1984), which is used for non-intrusive diagnosis and medical imaging. It qualitatively presents the change of sound resistance, which then allows us to distinguish different environments. The image's spatial resolution can be received by an array transducer (Macovski, 1979) and highly-converged, single-element transducers (Kino, 1987). Even though the quality can be worsened by uncertainties in amplitude and phase (Zhu \& Steinberg, 1993), B-mode is overall considered uncomplicated and reliable. However, due to its qualitative nature, medical diagnosis using these images is subjective and heavily based on the doctor's expertise.

A qualitative ultrasound technique (also known as tomographic ultrasound) is considered superior to B-mode in offering more valuable information (Jonathan \& Oelze, 2013). However, this method still downsides, including its limitation to only weak scattering media, high computation complexity, and commercial equipment capabilities. The application of this method is limited, mostly used with breast cancer.

Tomographic ultrasound is a technique based on inverse scattering theory. The DistortedBorn iterative method (DBIM) is often used to solve the inverse problem. In this method, Green's function is repeated each iteration, causing DBIM to converge quickly (Montero \& Janniel, 2009). A significant disadvantage of this approach is its divergence in strong scattering media. In reality, Born approximations hypothesize that the scattering pressure is so small that it can be neglected. It is only correct in weak scattering media. In stronger scattering media, Born approximation is not accurate anymore (Slaney et al., 1984). This problem can be solved by using multiple frequencies for reconstruction based on sound contrast (Haddadin \& Ebbini, 1997), (Haddadin \& Ebbini, 1998). In these studies, frequencies $\mathrm{f}_{1}$ and $\mathrm{f}_{2}$ are utilized to reconstruct the subject in $\mathrm{N}_{\mathrm{f} 1}$ and $\mathrm{N}_{\mathrm{f} 2}$ iterations. The low-frequency $f_{1}$ ensures the convergence of the algorithm to a contrast level near the true level at the cost of low spatial resolution. After that, the high-frequency $f_{2}$ can improve spatial resolution as maintaining the convergence. The reason behind this is the relatively small difference between the true contrast and initial contrast (Born approximation satisfied). In (Jonathan \& Oelze, 2013), (Tijhuis et al., 2001), (Lavarello \& Oelze, 2010), (Tran et al., 2016), the authors suggested using more than two frequencies to get the resolution of a tomographic ultrasound image closer to the level of tissue image reconstruction. Not only for ultrasound tomography, the multiple - frequency technique is also applied for ultrasound images in (Ma et al., 2015; Sayed, 2018; Varray et al., 2012; Yoshizumi et al., 2009). However, the use of different frequencies in different iterations is still inconsistent. Frequency hop is usually chosen based on the scenarios being simulated or tested, in fact. Using a multi-frequency approach, we proposed an effective method to increase tomographic ultrasound imaging quality with a fundamental tone and overtones (FTaOT). The fundamental tone is used in the first DBIM iteration, then its overtones for the next iterations. Numerous scenarios have been tested to prove the feasibility of our proposed method.

\section{MATERIALS AND METHOD}

\subsection{Distorted-Born Iterative Method}

We configured round DBIM measurements surrounding the subject. Transmitters, receivers can be randomly or pseudo-randomly arranged. The number of transducers are based on the scenario and 


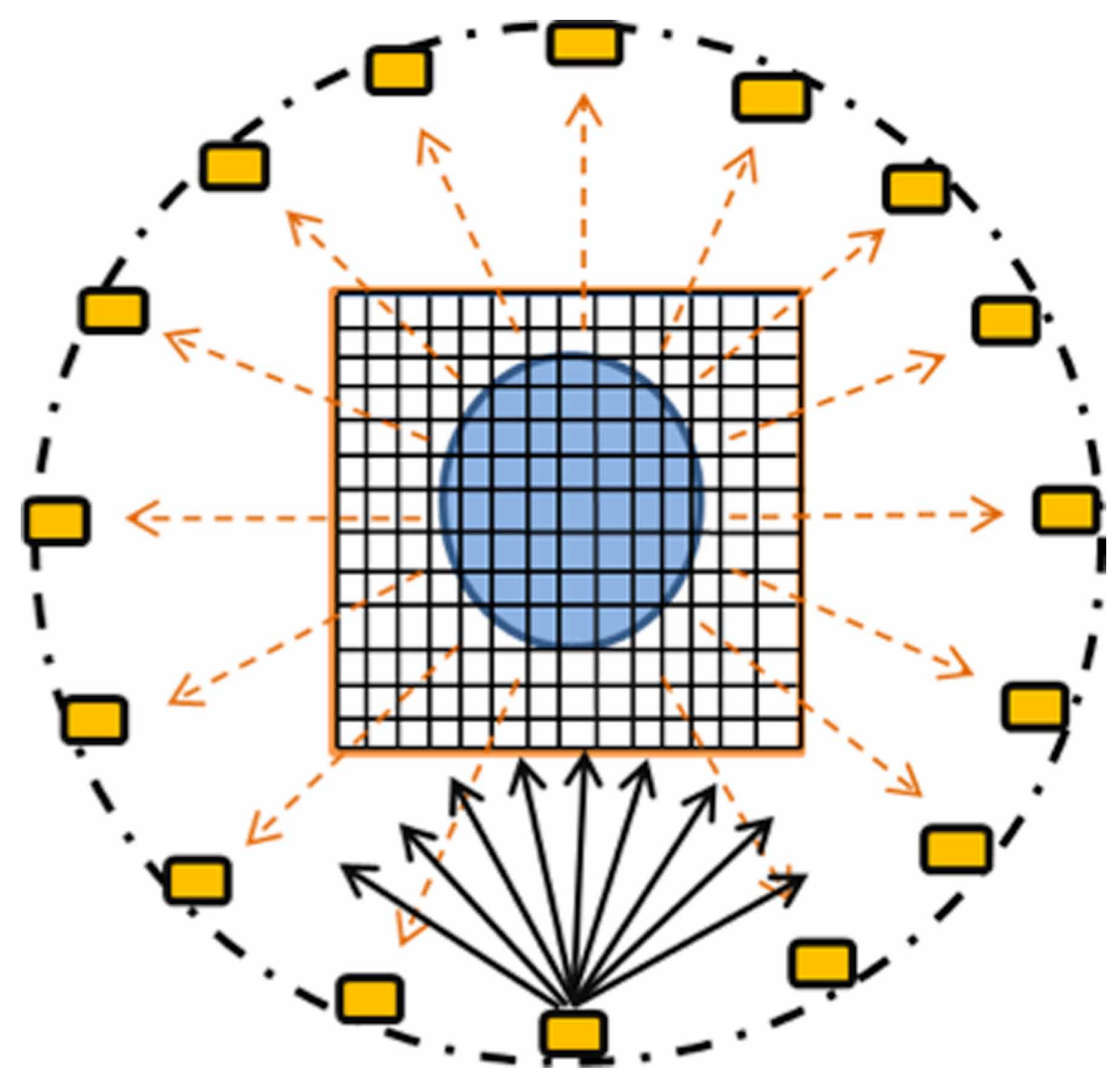

practical requirement. If there are too many transmitters and receivers, the measurement system would be more complicated and need more computation, storage, information processing, etc. It is also true vice versa. The locations of transmitters, receivers might be different or the same (with the current transducer technology, a transducer can both transmit and receive at a time).

Let there be $\mathrm{N}_{\mathrm{t}}$ transmitters and $\mathrm{N}_{\mathrm{r}}$ receivers. $\mathrm{N}_{\mathrm{t}}$ transmitters are set up at different angles around the subject to get sufficient data. The process is performed as follow: initially, the first transmitter sends ultrasound signal (others are inactive), all receivers are on and receive scattered ultrasound; as a result, we obtain a set of $\mathrm{N}_{\mathrm{r}}$ measurement from the first transmitter; afterward, we active the second transmitter and get another $\mathrm{N}_{\mathrm{r}}$ measurements with this device; etc. The process continues until the last transmitter is active and yields $\mathrm{N}_{t}$ sets of measurement values. With that, we then have sufficient data on the subject from different angles. Using only one transmitter at a time is equivalent to using a single transducer in tomographic ultrasound imaging.

Figure 1 shows the transmit/receive configuration of the tomographic ultrasound imaging system. The survey subject is a small-sized cylinder $\psi(\mathrm{r})$, being environment $\mathrm{A}_{1}$ positioned inside the background environment $\mathrm{A}_{2}$. Our target is to reconstruct the cylinder image, which is the region of interest (ROI) being divided into $\mathrm{N} \times \mathrm{N}$ squares (each square is one pixel). Suppose $\varrho_{0}$ is the unchanged background wavenumber in an infinite space containing a homogenous environment (like water). 
The subject also has unaltered density and wavenumber $\varrho$ (r) in space. The sound speed difference between the subject environment and background environment is called $\Delta u$. With the scattering media, as in Figure 1, the objective function can be calculated as follow:

$\psi(\vec{r})= \begin{cases}\varrho(\vec{r})^{2}-\varrho_{0}^{2}=\omega^{2}\left(\frac{1}{u_{1}^{2}}-\frac{1}{u_{0}^{2}}\right) & \text { if } \mathrm{r} \leq R \\ 0 & \text { if } \mathrm{r}>\mathrm{R}\end{cases}$

with $u_{1}$ and $u_{0}$ being sound speed in object and background environments, respectively; $\mathrm{f}$ being ultrasound frequency; $\omega$ being angular frequency ( $\omega=2 \pi \mathrm{f}$ ), and R being the object's radius. $\Delta u$ is a sound speed difference, also called sound contrast ( $\left.\Delta u=\frac{u_{1-u_{0}}}{u_{0}} \times 100 \%\right)$. Small $\Delta u$ means the scattering media is weak and vice versa.

The system's wave equation is shown below:

and the total sound pressure:

$$
\left(\nabla^{2}+\varrho_{0}^{2}(\vec{r})\right) \xi(\vec{r})=-\psi(\vec{r}) \xi(\vec{r})
$$

$$
\xi(\vec{r})=\xi^{i n c}(\vec{r})+\xi^{s c}(\vec{r})
$$

in which:

Scattered wave pressured is:

$$
\left.\xi^{s c}(\vec{r})=\iint \psi(\vec{r}) \xi(\vec{r}) G_{0}\left(\mid \vec{r}-\overrightarrow{r^{\prime}}\right)\right) d \overrightarrow{r^{\prime}}
$$

Incoming wave pressure is the zero-order Bessel Beam in two-dimension space:

$$
\underline{\xi}^{i n c}=J_{0}\left(\varrho_{0}\left|r-r_{k}\right|\right)
$$

with $\xi(\vec{r})$ being total sound pressure, $\xi^{\text {inc }}(\vec{r})$ being incoming wave pressure, $\xi^{s c}(\vec{r})$ being scattered wave pressure, $\mathrm{G}(\cdot)$ being Green's function, $J_{0}$ being zero-order Bessel function and $\left|r-r_{k}\right|$ being the distance between transmitter and the $\mathrm{k}^{\text {th }}$ point in ROI.

Bessel function $\left(\mathrm{J}_{0}\right)$ is often used in digital simulation as the transmitter signal (incoming wave, frequency f, wavelength $\lambda=u_{0} / f$, in which $u_{0}$ is the sound propagation speed in the background environment). When an ultrasound wave propagates in the environment: a) if the environment is homogeneous, the signal received is the incoming wave pressure; b) if the environment is nonhomogeneous (strange tumor exists), a few scenarios can happen when the incoming wave meets 
this subject: when the subject size is multiple times larger than incoming wave wavelength, the signal is reflected; when the subject size is equal or smaller than incoming wave wavelength, the ultrasound signal is scattered in every direction around the subject. It is taken into notice that ultrasound frequency used in clinical diagnosis is between $20 \mathrm{KHz}$ and $12 \mathrm{MHz}$. Therefore, if the sound the speed of the background environment is assumedly $1484 \mathrm{~m} / \mathrm{s}$, the wavelength is from $6.2 \mu \mathrm{m}$ to $74.2 \mathrm{~mm}$.

We use the method of moments (MoM) to discretize equation (4) by gridding the ROI as in Figure 1 and presented in the form of matrix. The pressure of ROI points can be calculated as follow:

$$
\underline{\xi}=(\underline{I}-\underline{C} \cdot D(\underline{\psi})) \xi^{i n c}
$$

The pressure of points outside ROI (scattering pressure) has the size of $N_{t} N_{r} \times 1$ :

$$
\xi^{s c}=\underline{B} \cdot D \cdot(\underline{\psi}) \cdot \underline{\xi}
$$

Utilizing Born approximation, we can find the linear connection between the scattering pressure difference $\Delta \xi^{s c}$ and the objective function $\Delta \underline{\psi}$ as follow:

$$
\Delta \xi^{s c}=(\underline{B} \cdot D \cdot(\underline{\psi})) \Delta \underline{\xi}=\underline{M} \cdot \Delta \underline{\psi}
$$

with system matrix $\underline{M}=\underline{B} . D(\underline{\xi}) ; \underline{B}$ being Green's function that represents the relation between the points and receivers; $\underline{C}$ being Green's function that describes the relation between points; $\underline{I}$ being identity matrix; $D(\cdot)$ is an operator that transforms a vector into a diagonal matrix (Montero \& Janniel, 2009).

With one transmitter and one receiver, we form a matrix $\underline{M}$ and a vector quantity $\Delta \xi^{s c}$. The unknown vector $\psi$ has $\mathrm{N} \times \mathrm{N}$ variables, with the number of variables is the same as the number of ROI pixels. We can calculate the objective function as follow:

$$
\underline{\psi}^{n}=\underline{\psi}^{n-1}+\Delta \underline{\psi}^{n-1}
$$

with $\psi^{n}$ and $\psi^{n-1}$ being the current and the previous objective function. The data is processed with DBIM to recover sound contrast. By this way, we can detect if there is a tumor in the environment. DBIM utilizes Born approximation to calculate in iterations of inverse scattering problems.

Tikhonov method is used to solve the uncertainty system of equations to calculate $\Delta \underline{\psi}$ :

$$
\Delta \underline{\psi}=\arg \min _{\Delta \underline{\psi}}\left\|\Delta \xi^{s c}-\underline{M_{t}} \Delta \underline{\psi}\right\|_{2}^{2}+\gamma\|\Delta \underline{\psi}\|_{2}^{2}
$$

in which $\underline{M_{t}}$ is the matrix $\mathrm{N}_{\mathrm{t}} \mathrm{N}_{\mathrm{r}} \times \mathrm{N}^{2}$ created by $\mathrm{N}_{\mathrm{t}} \mathrm{N}_{\mathrm{r}}$ measurements.

\subsection{Inverse scattering problem in DBIM.}

To solve the inverse problem when there is interference, we use the nonlinear conjugate gradient method (NCG) (Golub et al., 1999). NCG in Algorithm 1 is utilized to solve equation (10): 


$$
\begin{aligned}
& \text { Algorithm 1: Nonlinear Conjugate Gradient Method (NCG) } \\
& \text { 1. Initialize } \Delta \psi \text { in the form of vector } 0 \\
& \text { 2. Initialize } \underline{b}_{(0)}=\underline{M}_{t}^{H} \cdot \Delta \underline{\xi}_{t}^{s c} \\
& \text { 3. Initialize } \underline{x}_{(0)}=\underline{b}_{(0)} \text { and } \underline{r}_{(0)}=\underline{b}_{(0)} \\
& \text { 4. for } n=1 \text { to the largest value, do } \\
& \text { 5. } \underline{q}_{(n)}=\underline{M}_{t} \cdot \underline{x}_{(n-1)} \\
& \text { 6. } \quad \propto_{(n)}=\frac{\underline{r}_{(n-1)}^{H} \cdot \underline{r}_{(n-1)}}{\underline{q}_{(n)}^{H} \underline{q}_{(n)}+\gamma \underline{x}_{(n-1)}^{H} \cdot \underline{x}_{(n-1)}} \\
& \text { 7. } \quad \underline{s}_{(n)}=\underline{M}_{t}^{H} \cdot \underline{q}_{(n)} \\
& \text { 8. } \quad \underline{r}_{(n)}=\underline{r}_{(n-1)}-\propto_{(n)}\left(\underline{s}_{(n)}+\gamma \underline{x}_{(n-1)}\right) \\
& \text { 9. } \beta_{(n)}=\frac{\underline{r}_{(n)}^{H} \cdot \underline{r}_{(n)}}{\underline{r}_{(n-1)}^{H} \cdot \underline{r}_{(n-1)}} \\
& \text { 10. } \Delta \underline{\psi}_{(n)}=\Delta \underline{\psi}_{(n-1)} \propto_{(n)} \underline{x}_{(n-1)} \\
& \text { 11. } \underline{x}_{(n)}=\underline{r}_{(n)}+\beta_{(n)} \underline{x}_{(n-1)} \\
& \text { 12. if }\|\underline{r}\|<\text { tolerance, then } \\
& \text { 13. Break iteration } \\
& \text { 14. end if } \\
& \text { 15. end for } \\
& \text { To choose } \gamma \text {, we use: } \\
& \gamma=0.5 \sigma_{0}^{2} \max \left\{10^{R R E}, 10^{-4}\right\}
\end{aligned}
$$

in which, $\sigma_{0}^{2}$ is calculated using the exponent iteration method with Rayleigh quotient as in Algorithm 2 (see Fig. 2).

\section{PROPOSED METHOD}

\subsection{Initial Frequency and Frequency Hop}

If the low frequency is used in the imaging process, we will get a poor spatial resolution, yet the convergence speed is good. On the contrary, if high frequency is used, the spatial resolution is good, at the cost of convergence speed. Therefore, using multiple frequencies in different iterations is necessary to achieve a recovered image with high convergence speed and spatial resolution. Simulation scenarios designer usually chooses frequency hop. In our study, we choose the initial frequency using Born approximation, with $f=f_{1}$ so that:

$$
\Delta \boldsymbol{\varphi}=\mathbf{2} \boldsymbol{\omega}\left(\frac{1}{u}-\frac{1}{u_{0}}\right) \boldsymbol{R}<\boldsymbol{\pi}
$$


Figure 2. Transmit/receive configuration of the tomographic ultrasound imaging system
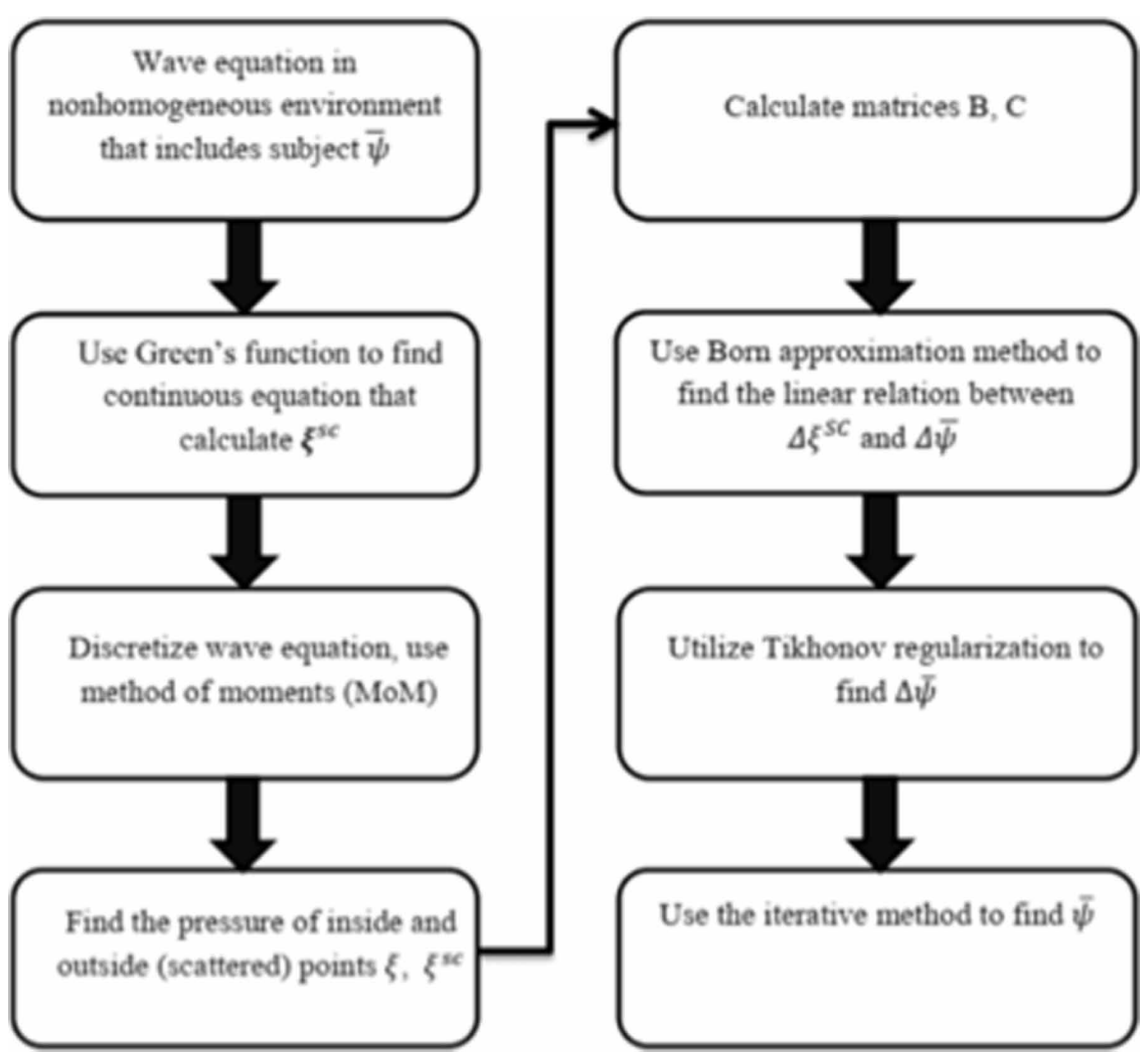

with $f_{1}$ we recover the image at contrast $u_{1}$. Afterward, choose the frequency hop value and find $f_{2}$. We then recover the image at contrast $u_{2}$ updated from $u_{1}$. Continue to do so until we reach the targeted contrast $\mathrm{u}^{*}\left(u_{1}<u_{2}<\ldots<\mathrm{u}^{*}\right)$. The importance of increasing frequency as such is that it gradually improves the resolution and approaches image reconstruction at the tissue level.

\subsection{Proposed Image Reconstruction Method Using DBIM}

Figure 3 presents the flowchart of the proposed method. At first initialize $\underline{\psi}_{(n)}=\underline{\psi}_{(0)}$ and $\underline{\xi}_{0}=\underline{\xi}^{\text {inc }}$ with $n=0$. Assume that we have $N_{t}$ transmitters and $N_{r}$ receivers, pick $N_{t}$ and $N_{r}$ that satisfy $N_{t} \times N_{r} \approx N^{2}$. With $n=1$, run DBIM using $f_{1}$, which is equal to $f_{0}$ ( $f_{0}$ is the fundamental tone frequency) in the first iteration of DBIM. The recovered image at this step has a contrast level of $\mathrm{u}_{1}$. Similarly, with $n=2$, run DBIM with $f_{2}=2 f_{0}$ and update contrast $\mathrm{u}_{1}$ to $\mathrm{u}_{2}$. When more than two frequencies are used in the survey, run DBIM with $n=n+1$, the method used $f_{n}=n f_{0}\left(f_{n}\right.$ 
is the $\mathrm{n}^{\text {th }}$ overtone), which yields clearer spatial resolution gradually. If the condition $n \leq n_{\text {iter }}$ is satisfied, continuing to the next iteration. End the algorithm if this condition is satisfied.

The process of DBIM is presented in Algorithm 3 below:

Algorithm 3. Distorted-Born Iteration Method

1. Choose initial values: $\underline{\psi}_{(n)}=\underline{\psi}_{(0)}$ and $\underline{\xi}_{0}=\underline{\xi}^{i n c}$ using (5)

For $n=1$ to $N_{D B I M}$, do

2. Calculate $\underline{B}$ và $\underline{C}$

3. Calculate $\underline{\xi}, \xi^{s c}$ in accordance with $\underline{\psi}_{(n)}$ using $(6,7)$

4. Calculate $\Delta \xi^{s c}$ using (8)

5. Calculate $\Delta \underline{\psi}_{(n)}$ using Nonlinear Conjugate Gradient Method (10)

6. Calculate $\underline{\psi}^{n}=\underline{\psi}^{n-1}+\Delta \underline{\psi}^{n-1}$

End For

\subsection{Distorted-Born Iteration Method Using Fundamental Tone and Overtones (FTaOT-DBIM)}

In physics, the lowest frequency $\left(\mathrm{f}_{0}\right)$ is called fundamental tone while the higher ones $\left(2 \mathrm{f}_{0}, 3 \mathrm{f}_{0}, 4 \mathrm{f}_{0}\right.$, $\left.5 f_{0}, \ldots\right)$ are called overtones. It is the fact that when an oscillator generates frequency $f$ (used for image reconstruction and we call it fundamental tone), then it also generates overtones ( $2 \mathrm{f}, 3 \mathrm{f}, 4 \mathrm{f}, \ldots)$. We utilize the fundamental tone and its overtones to penetrate the subject and progressively increase image resolution. Therefore, in our study, frequency $\mathrm{f}$ is used in the first iteration, and naturally, its overtones are applied in the following iterations. By that, the resolution is increased progressively along with the reconstruction. We then have:

$\mathrm{N}_{\text {iter }}=\mathrm{N}_{\mathrm{f} 1}+\mathrm{N}_{\mathrm{f} 2}+\mathrm{N}_{\mathrm{f} 3}+\mathrm{N}_{\mathrm{f} 4}+\mathrm{N}_{\mathrm{f} 5}+\mathrm{N}_{\mathrm{f} 6}+\mathrm{N}_{\mathrm{f} 7}+\mathrm{N}_{\mathrm{f} 8}$

The FTaOT-DBIM is presented in Algorithm 4. In which, $\mathrm{N}_{\mathrm{f} 1}=\mathrm{N}_{\mathrm{f} 2}=\mathrm{N}_{\mathrm{f} 3}=\mathrm{N}_{\mathrm{f} 4}=\mathrm{N}_{\mathrm{f5}} \mathrm{N}_{\mathrm{f} 6}=\mathrm{N}_{\mathrm{f} 7}$ $=\mathrm{N}_{\mathrm{f} 8}=1$.

Algorithm 4. The FTaOT-DBIM

1. Choose the initial values: $\underline{\psi}_{(n)}=\underline{\psi}_{(0)} ; \underline{\xi}_{0}=\underline{\xi}^{i n c}$ using (5)

2. If $n=0$, do Algorithm 3, $f=0$. End for

3. If $n=n+1$ to $\mathrm{N}_{f 1}$, do Algorithm 3, using $f_{1}$. End for.

4. If $n=\mathrm{n}+1$ to $\mathrm{N}_{\mathrm{f} 2}$, do Algorithm 3, using $\mathrm{f}_{2}$. End for.

5. If $n=\mathrm{n}+1$ to $\mathrm{N}_{\mathrm{f3}}$, do Algorithm 3, using $\mathrm{f}_{3}$. End for.

6. If $\mathrm{n}=\mathrm{n}+1$ to $\mathrm{N}_{\mathrm{f4}}$, do Algorithm 3, using $\mathrm{f}_{4}$. End for.

7. If $n=\mathrm{n}+1$ to $\mathrm{N}_{\mathrm{f5}}$, do Algorithm 3, using $\mathrm{f}_{5}$. End for.

8. If $n=\mathrm{n}+1$ to $\mathrm{N}_{\mathrm{f} 6}$, do Algorithm 3, using $\mathrm{f}_{6}$. End for.

9. If $n=\mathrm{n}+1$ to $\mathrm{N}_{\mathrm{f} 7}$, do Algorithm 3, using $\mathrm{f}_{7}$. End for.

10. If $n=\mathrm{n}+1$ to $\mathrm{N}_{\mathrm{f} 8}$, do Algorithm 3, using $\mathrm{f}_{8}$. End for.

11. Calculate RRE using (13)

In which, relative residual error (RRE) is calculated as below:

$R R E=\sum_{i=1}^{N} \sum_{j=1}^{N} \frac{\left|C_{i j}-\hat{C}_{i j}\right|}{C_{i j}}$ 
Figure 3. Flowchart of the DBIM using fundamental tone and overtones (FTaOT)

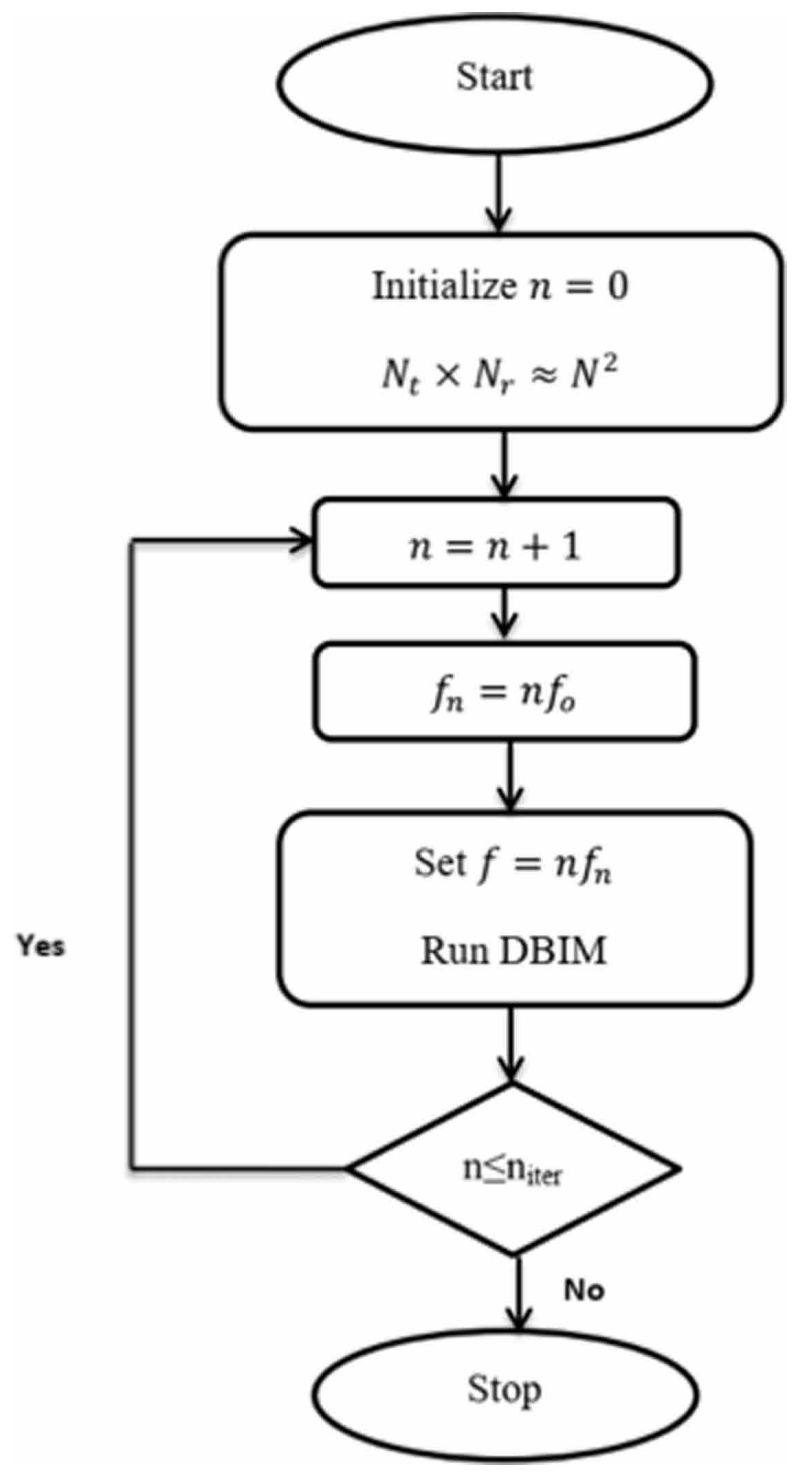

\section{RESULTS AND DISCUSSIONS}

Simulation parameters: Frequencies $\mathrm{f}_{1}=1 \mathrm{MHz}, \mathrm{f}_{2}=2 \mathrm{MHz}, \mathrm{f}_{3}=3 \mathrm{MHz}, \mathrm{f}_{4}=4 \mathrm{MHz}, \mathrm{f}_{5}=5 \mathrm{MHz}$, $\mathrm{f}_{6}=6 \mathrm{MHz}, \mathrm{f}_{7}=7 \mathrm{MHz}, \mathrm{f}_{8}=8 \mathrm{MHz}$; Number of pixels $\mathrm{N}=20$; Number of transmitters $\mathrm{N}_{\mathrm{t}}=11$; Number of receivers $\mathrm{N}_{\mathrm{r}}=22$; Number of iterations $\mathrm{N}_{\text {sum }}=8$; Scattering media diameter $7.3 \mathrm{~mm}$; Sound contrast 30\%; Gaussian noise 10\%; Distance from the transmitters and receivers to the subject center are $50 \mathrm{~mm}$ và $60 \mathrm{~mm}$, respectively.

\subsection{The Proposed FTaOT-DBIM Simulation Results}

Figure 4 shows the ideal objective function that the ultrasound imaging system needs to recover. Figure 5 presents the error performance of the proposed method in comparison with the conventional ones. It shows that the normalized error is significantly reduced compared to the conventional methods. 


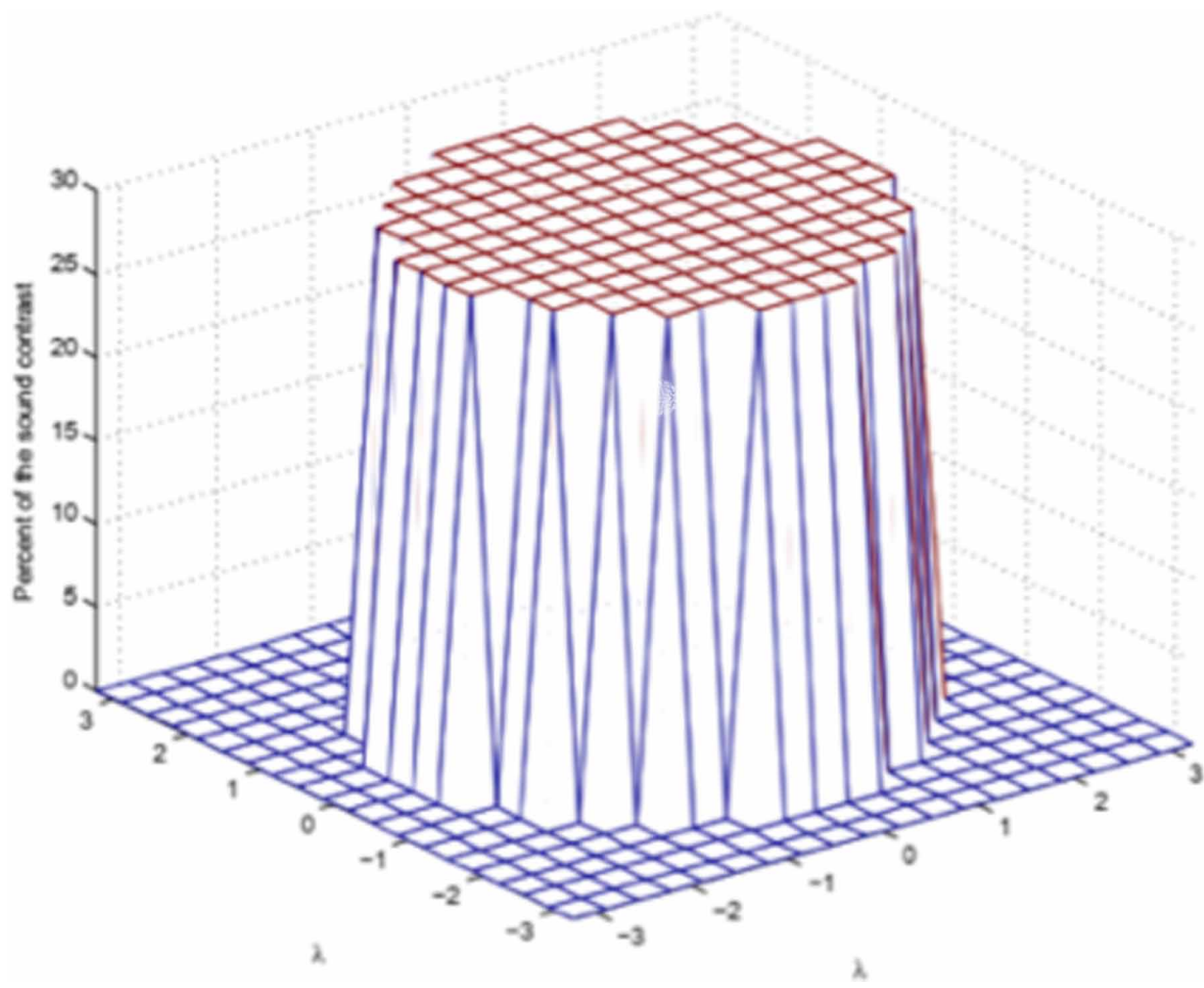

After eight iterations, the normalized errors of the DBIM, DF-DBIM, and FTaOT methods are 0.4205, 0.1293 , and 0.0709 , respectively. Therefore, the proposed method's normalized error is $45 \%$ reduced compared to the conventional DF-DBIM method.

Figure 6 shows the recovered results for the DBIM, DF-DBIM, and FTaOT-DBIM methods after the $\mathrm{N}_{\text {sum }}$ iteration. Visually, we can see that the background noise in the proposed method is lower than the traditional methods. The results recovered by the proposed method are closer to the ideal objective function than the conventional methods.

Figure 7 shows the imaging runtime comparison between FTaOT-DBIM and SF-DBIM (single frequency DBIM). It can be seen that the FTaOT-DBIM takes more time; however, the difference is insignificant. 
Figure 5. The error performance of the proposed method in comparison with the conventional ones

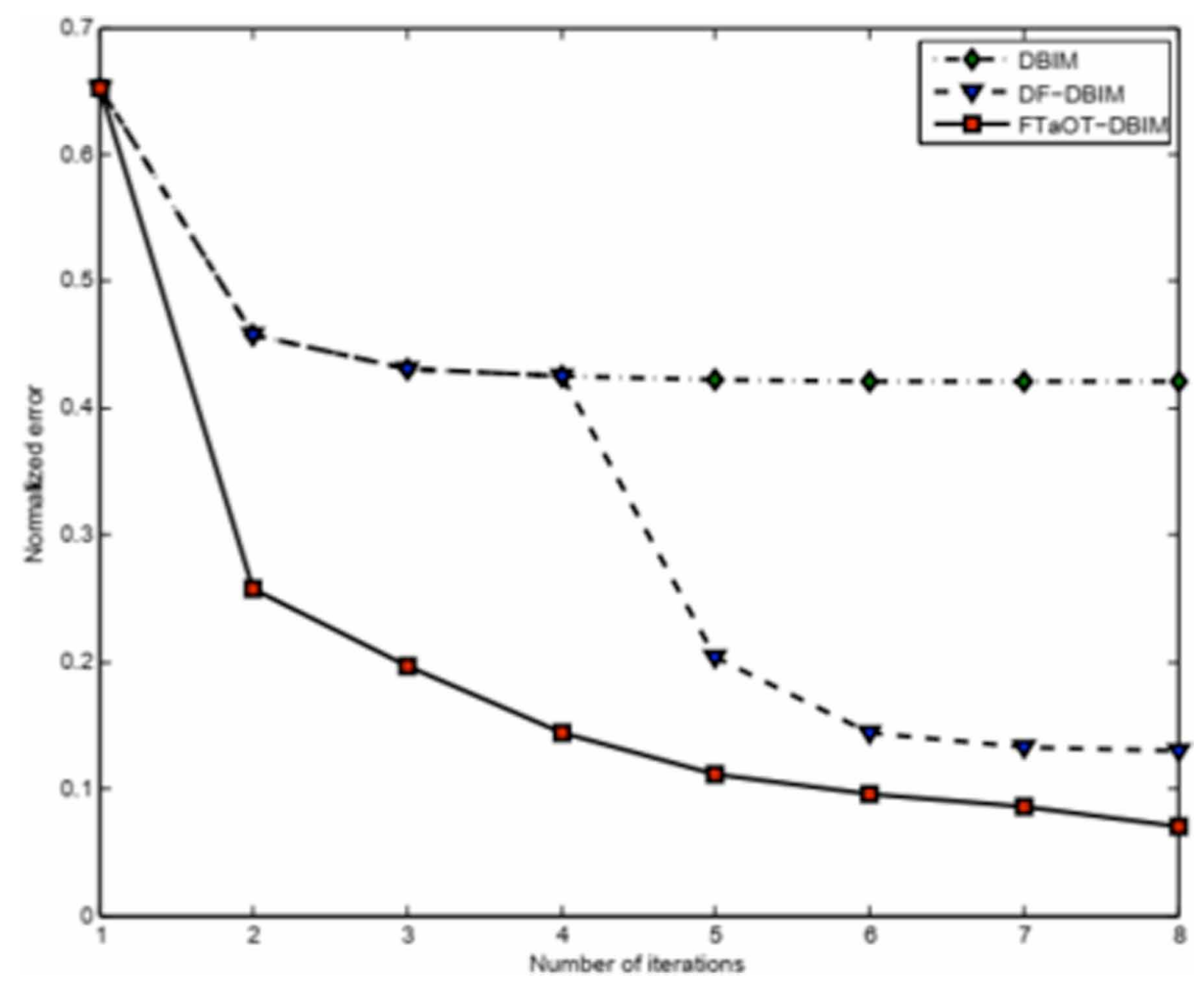

\subsection{Assessment of the Proposed Method and Multi- Frequency-Based Different Approaches}

The different scenarios of the DBIM using multi-frequency information are shown in Tables 1-10.

The normalized errors of the scenarios are shown in Table 11. It can be seen that scenario 22 offers the smallest error $(\mathrm{RRE}=0.0757)$. It is still larger than the error of the proposed method (RRE $=0.0709$ ). Therefore, the proposed method is a robust approach for enhanced reconstruction quality of ultrasound tomography based on fundamental tone and overtones.

\section{DISCUSSION}

Our study uses a fundamental tone for the first iteration in DBIM, then consecutively uses its overtones for the next ones. The ratio between the starting and the final frequency is varied due to various kinds of simulation scenarios. The simulation results proved that multi-frequency ultrasound tomography is capable of improving the quality of the image. Fig. 5 also confirmed that using multi-frequency DBIM image reconstruction offers better performance than conventional DBIM and our previous study (Tran et al., 2016). The technique of using multi-frequency is realistic because the ultrasound probes now a large frequency bandwidth.

In our work, the starting frequency is chosen at a relatively low value compared to (Haddadin \& Ebbini, 1997; Haddadin \& Ebbini, 1998; Lavarello \& Oelze, 2010; Tijhuis et al., 2001) to ensure 
Figure 6. The recovered results for the DBIM, DF-DBIM, and FTaOT-DBIM methods after the $\mathrm{N}_{\text {sum }}$ iteration
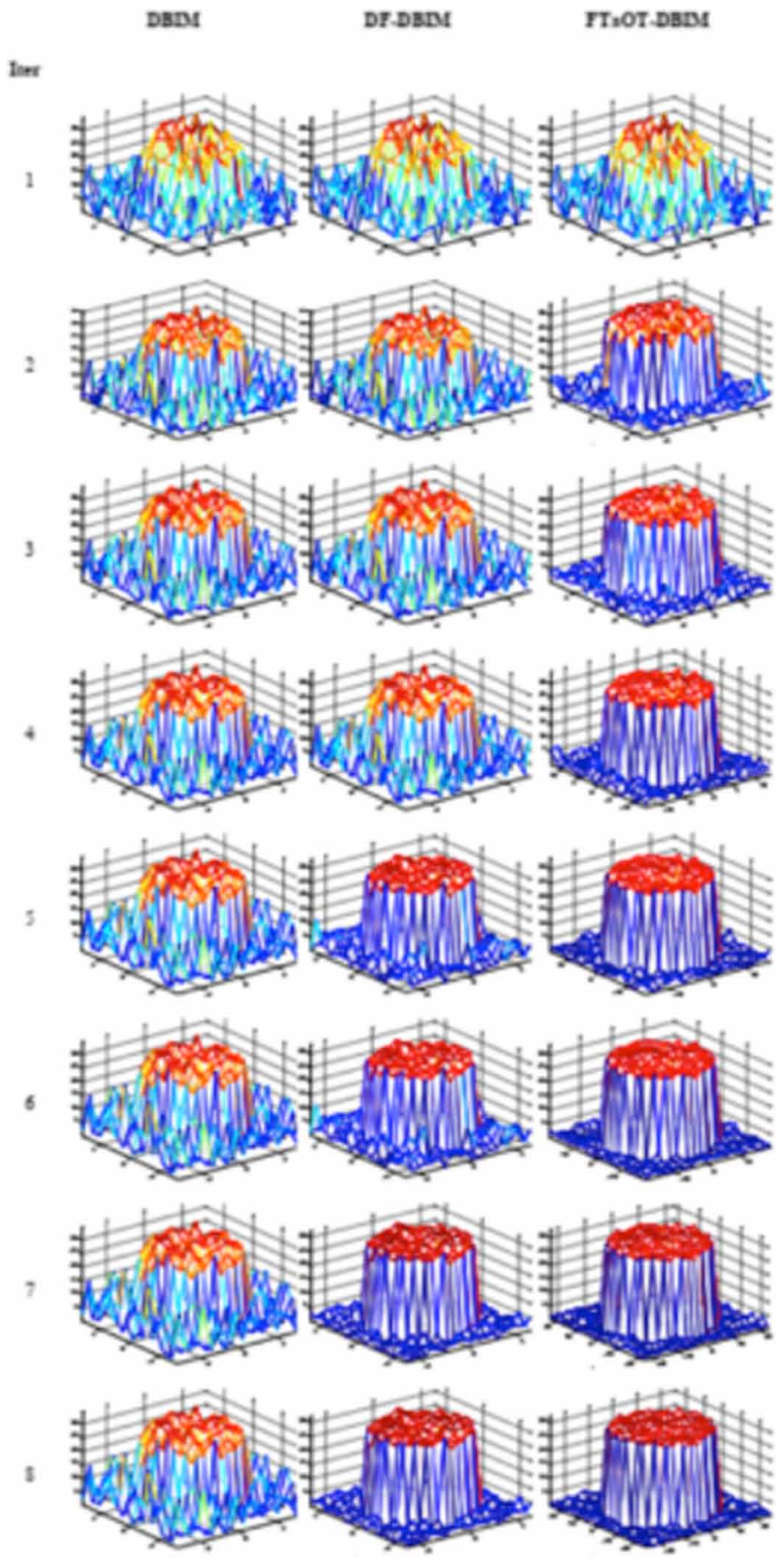
Figure 7. The imaging runtime comparison between FTaOT-DBIM and SF-DBIM (single frequency DBIM)

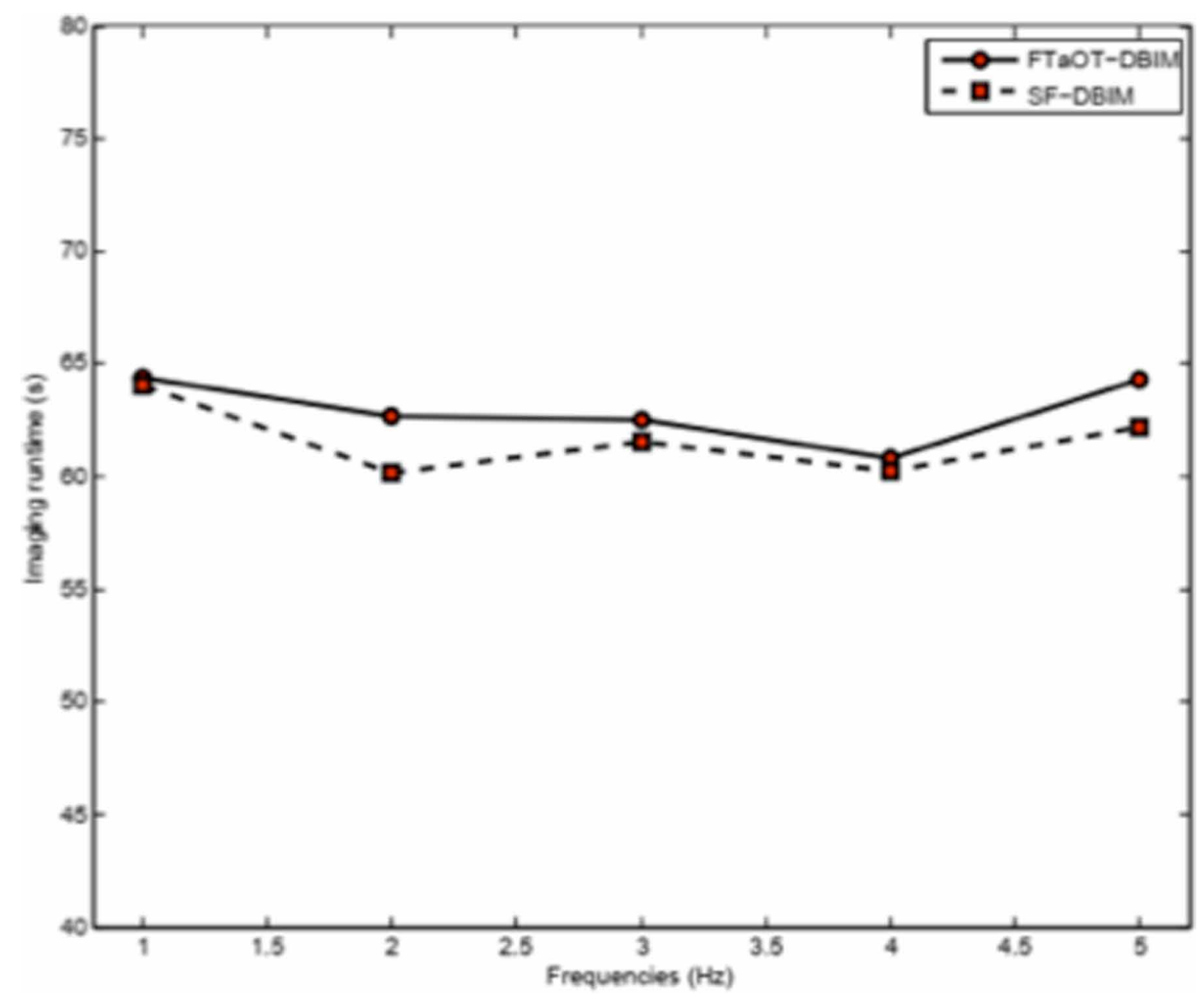

convergence. After that, we use its overtones for the next ones to archive the good spatial resolution. It is noteworthy that the estimated images in Fig.6 contain many interesting features. It strongly confirms the effectiveness of FTaOT-DBIM with DBIM and DF-DBIM.

While there are significant findings reported in our study, the study itself suffers from certain limitations. Firstly, Fig. 5 shows that after the 6th iteration, the normalized error of FTaOT-DBIM reaches a floor. We can reduce this floor by reducing the tolerated reconstruction error in the reconstructed algorithm. It will lead to a more complex computational procedure and, as a consequence, a much longer imaging time. Secondly, this research is only concerned with numerical simulation. Further investigations are required for extracting the valuable information from the multi-frequency ultrasound images. It may be translated to diagnostic ultrasound imaging. 
Table 1. DBIM Using Multi-Frequency in the case of different $N_{f 1} s$

\begin{tabular}{|l|l|l|l|l|l|l|l|l|}
\hline Iterations & $\mathbf{1}$ & $\mathbf{2}$ & $\mathbf{3}$ & $\mathbf{4}$ & $\mathbf{5}$ & $\mathbf{6}$ & $\mathbf{7}$ & $\mathbf{8}$ \\
\hline Scenario 1 & $\mathrm{f}_{1}$ & $\mathrm{f}_{2}$ & $\mathrm{f}_{3}$ & $\mathrm{f}_{4}$ & $\mathrm{f}_{5}$ & $\mathrm{f}_{6}$ & $\mathrm{f}_{7}$ \\
\hline Scenario 2 & $\mathrm{f}_{1}$ & $\mathrm{f}_{2}$ & $\mathrm{f}_{3}$ & $\mathrm{f}_{4}$ & $\mathrm{f}_{5}$ & $\mathrm{f}_{6}$ \\
\hline Scenario 3 & $\mathrm{f}_{1}$ & & $\mathrm{f}_{2}$ & $\mathrm{f}_{3}$ & $\mathrm{f}_{4}$ & $\mathrm{f}_{5}$ \\
\hline Scenario 4 & $\mathrm{f}_{1}$ & & & $\mathrm{f}_{2}$ & $\mathrm{f}_{3}$ & $\mathrm{f}_{4}$ \\
\hline Scenario 5 & $\mathrm{f}_{1}$ & & & & $\mathrm{f}_{2}$ & $\mathrm{f}_{3}$ \\
\hline
\end{tabular}

Table 2. DBIM Using Multi-Frequency in the case of different $N_{f 2} s$

\begin{tabular}{|l|l|l|l|l|l|l|l|l|}
\hline Iterations & $\mathbf{1}$ & $\mathbf{2}$ & $\mathbf{3}$ & $\mathbf{4}$ & $\mathbf{5}$ & $\mathbf{6}$ & $\mathbf{7}$ & $\mathbf{8}$ \\
\hline Scenario 6 & $\mathrm{f}_{1}$ & $\mathrm{f}_{2}$ & $\mathrm{f}_{3}$ & $\mathrm{f}_{4}$ & $\mathrm{f}_{5}$ & $\mathrm{f}_{6}$ & $\mathrm{f}_{7}$ \\
\hline Scenario 7 & $\mathrm{f}_{1}$ & $\mathrm{f}_{2}$ & $\mathrm{f}_{3}$ & $\mathrm{f}_{4}$ & $\mathrm{f}_{5}$ & $\mathrm{f}_{6}$ \\
\hline Scenario 8 & $\mathrm{f}_{1}$ & $\mathrm{f}_{2}$ & & $\mathrm{f}_{3}$ & $\mathrm{f}_{4}$ & $\mathrm{f}_{5}$ \\
\hline Scenario 9 & $\mathrm{f}_{1}$ & $\mathrm{f}_{2}$ & & & $\mathrm{f}_{3}$ & $\mathrm{f}_{4}$ \\
\hline Scenario 10 & $\mathrm{f}_{1}$ & $\mathrm{f}_{2}$ & & & & $\mathrm{f}_{3}$ \\
\hline
\end{tabular}

Table 3. DBIM Using Multi-Frequency in the case of different $N_{f 3} S$

\begin{tabular}{|l|l|l|l|l|l|l|l|l|}
\hline Iterations & $\mathbf{1}$ & $\mathbf{2}$ & $\mathbf{3}$ & $\mathbf{4}$ & $\mathbf{5}$ & $\mathbf{6}$ & $\mathbf{7}$ & $\mathbf{8}$ \\
\hline Scenario 11 & $\mathrm{f}_{1}$ & $\mathrm{f}_{2}$ & $\mathrm{f}_{3}$ & $\mathrm{f}_{4}$ & $\mathrm{f}_{5}$ & $\mathrm{f}_{6}$ & $\mathrm{f}_{7}$ \\
\hline Scenario 12 & $\mathrm{f}_{1}$ & $\mathrm{f}_{2}$ & $\mathrm{f}_{3}$ & & $\mathrm{f}_{4}$ & $\mathrm{f}_{5}$ & $\mathrm{f}_{6}$ \\
\hline Scenario 13 & $\mathrm{f}_{1}$ & $\mathrm{f}_{2}$ & $\mathrm{f}_{3}$ & & & $\mathrm{f}_{4}$ & $\mathrm{f}_{5}$ \\
\hline Scenario 14 & $\mathrm{f}_{1}$ & $\mathrm{f}_{2}$ & $\mathrm{f}_{3}$ & & $\mathrm{f}_{4}$ \\
\hline Scenario 15 & $\mathrm{f}_{1}$ & $\mathrm{f}_{2}$ & $\mathrm{f}_{3}$ & & & & \\
\hline
\end{tabular}

Table 4. DBIM Using Multi-Frequency in the case of different $N_{f 4} s$

\begin{tabular}{|l|l|l|l|l|l|l|l|l|}
\hline Iterations & $\mathbf{1}$ & $\mathbf{2}$ & $\mathbf{3}$ & $\mathbf{4}$ & $\mathbf{5}$ & $\mathbf{6}$ & $\mathbf{7}$ & $\mathbf{8}$ \\
\hline Scenario 16 & $\mathrm{f}_{1}$ & $\mathrm{f}_{2}$ & $\mathrm{f}_{3}$ & $\mathrm{f}_{4}$ & $\mathrm{f}_{5}$ & $\mathrm{f}_{6}$ & $\mathrm{f}_{7}$ \\
\hline Scenario 17 & $\mathrm{f}_{1}$ & $\mathrm{f}_{2}$ & $\mathrm{f}_{3}$ & $\mathrm{f}_{4}$ & & $\mathrm{f}_{5}$ & $\mathrm{f}_{6}$ \\
\hline Scenario 18 & $\mathrm{f}_{1}$ & $\mathrm{f}_{2}$ & $\mathrm{f}_{3}$ & $\mathrm{f}_{4}$ & & & $\mathrm{f}_{5}$ \\
\hline Scenario 19 & $\mathrm{f}_{1}$ & $\mathrm{f}_{2}$ & $\mathrm{f}_{3}$ & $\mathrm{f}_{4}$ & & & & \\
\hline
\end{tabular}


Table 5. DBIM Using Multi-Frequency in the case of different $\mathrm{N}_{\mathrm{f5}} \mathrm{s}$

\begin{tabular}{|l|l|l|l|l|l|l|l|l|}
\hline Iterations & $\mathbf{1}$ & $\mathbf{2}$ & $\mathbf{3}$ & $\mathbf{4}$ & $\mathbf{5}$ & $\mathbf{6}$ & $\mathbf{7}$ & $\mathbf{8}$ \\
\hline Scenario 20 & $\mathrm{f}_{1}$ & $\mathrm{f}_{2}$ & $\mathrm{f}_{3}$ & $\mathrm{f}_{4}$ & $\mathrm{f}_{5}$ & $\mathrm{f}_{6}$ & $\mathrm{f}_{7}$ \\
\hline Scenario 21 & $\mathrm{f}_{1}$ & $\mathrm{f}_{2}$ & $\mathrm{f}_{3}$ & $\mathrm{f}_{4}$ & $\mathrm{f}_{5}$ & & $\mathrm{f}_{6}$ \\
\hline Scenario 22 & $\mathrm{f}_{1}$ & $\mathrm{f}_{2}$ & $\mathrm{f}_{3}$ & $\mathrm{f}_{4}$ & $\mathrm{f}_{5}$ & & \\
\hline
\end{tabular}

Table 6. DBIM Using Multi-Frequency in the case of different $\mathrm{N}_{\mathrm{f} 6} \mathrm{~s}$

\begin{tabular}{|l|l|l|l|l|l|l|l|l|}
\hline Iterations & $\mathbf{1}$ & $\mathbf{2}$ & $\mathbf{3}$ & $\mathbf{4}$ & $\mathbf{5}$ & $\mathbf{6}$ & $\mathbf{7}$ & $\mathbf{8}$ \\
\hline Scenario 23 & $\mathrm{f}_{1}$ & $\mathrm{f}_{2}$ & $\mathrm{f}_{3}$ & $\mathrm{f}_{4}$ & $\mathrm{f}_{5}$ & $\mathrm{f}_{6}$ & & $\mathrm{f}_{7}$ \\
\hline Scenario 24 & $\mathrm{f}_{1}$ & $\mathrm{f}_{2}$ & $\mathrm{f}_{3}$ & $\mathrm{f}_{4}$ & $\mathrm{f}_{5}$ & $\mathrm{f}_{6}$ & & \\
\hline
\end{tabular}

Table 7. DBIM Using Multi-Frequency in the case of different $N_{f 1} s$ and $N_{f 2} s$

\begin{tabular}{|l|l|l|l|l|l|l|l|l|}
\hline Iterations & $\mathbf{1}$ & $\mathbf{2}$ & $\mathbf{3}$ & $\mathbf{4}$ & $\mathbf{5}$ & $\mathbf{6}$ & $\mathbf{7}$ & $\mathbf{8}$ \\
\hline Scenario 25 & $\mathrm{f}_{1}$ & $\mathrm{f}_{2}$ & $\mathrm{f}_{3}$ & $\mathrm{f}_{4}$ & $\mathrm{f}_{5}$ & $\mathrm{f}_{6}$ \\
\hline Scenario 26 & $\mathrm{f}_{1}$ & $\mathrm{f}_{2}$ & $\mathrm{f}_{3}$ & $\mathrm{f}_{4}$ & $\mathrm{f}_{5}$ \\
\hline Scenario 27 & $\mathrm{f}_{1}$ & $\mathrm{f}_{2}$ & & $\mathrm{f}_{3}$ & $\mathrm{f}_{4}$ \\
\hline Scenario 28 & $\mathrm{f}_{1}$ & $\mathrm{f}_{2}$ & & & $\mathrm{f}_{3}$ \\
\hline Scenario 29 & $\mathrm{f}_{1}$ & $\mathrm{f}_{2}$ & $\mathrm{f}_{3}$ & $\mathrm{f}_{4}$ & $\mathrm{f}_{5}$ \\
\hline Scenario 30 & $\mathrm{f}_{1}$ & $\mathrm{f}_{2}$ & & $\mathrm{f}_{3}$ & $\mathrm{f}_{4}$ \\
\hline Scenario 31 & $\mathrm{f}_{1}$ & $\mathrm{f}_{2}$ & & & $\mathrm{f}_{3}$ \\
\hline
\end{tabular}

Table 8. DBIM Using Multi-Frequency in the case of different $N_{f 2} s$ and $N_{f 3} s$

\begin{tabular}{|l|l|l|l|l|l|l|l|l|}
\hline Iterations & $\mathbf{1}$ & $\mathbf{2}$ & $\mathbf{3}$ & $\mathbf{4}$ & $\mathbf{5}$ & $\mathbf{6}$ & $\mathbf{7}$ & $\mathbf{8}$ \\
\hline Scenario 32 & $\mathrm{f}_{1}$ & $\mathrm{f}_{2}$ & $\mathrm{f}_{3}$ & $\mathrm{f}_{4}$ & $\mathrm{f}_{5}$ & $\mathrm{f}_{6}$ \\
\hline Scenario 33 & $\mathrm{f}_{1}$ & $\mathrm{f}_{2}$ & $\mathrm{f}_{3}$ & $\mathrm{f}_{4}$ & $\mathrm{f}_{5}$ \\
\hline Scenario 34 & $\mathrm{f}_{1}$ & $\mathrm{f}_{2}$ & $\mathrm{f}_{3}$ & $\mathrm{f}_{4}$ \\
\hline Scenario 35 & $\mathrm{f}_{1}$ & $\mathrm{f}_{2}$ & $\mathrm{f}_{3}$ & \multicolumn{5}{l|}{} \\
\hline Scenario 36 & $\mathrm{f}_{1}$ & $\mathrm{f}_{2}$ & $\mathrm{f}_{3}$ & $\mathrm{f}_{4}$ & $\mathrm{f}_{5}$ \\
\hline Scenario 37 & $\mathrm{f}_{1}$ & $\mathrm{f}_{2}$ & $\mathrm{f}_{3}$ & $\mathrm{f}_{4}$ \\
\hline Scenario 38 & $\mathrm{f}_{1}$ & $\mathrm{f}_{2}$ & $\mathrm{f}_{3}$ & \multicolumn{5}{l|}{} \\
\hline
\end{tabular}


Table 9. DBIM Using Multi-Frequency in case of different $N_{f 1} s, N_{f 2} s$, and $N_{f 3} s$

\begin{tabular}{|c|c|c|c|c|c|c|c|c|}
\hline Iterations & 1 & 2 & 3 & 4 & 5 & 6 & 7 & \multirow{3}{*}{$\frac{\mathbf{8}}{\mathrm{f}_{5}}$} \\
\hline Scenario 39 & \multicolumn{2}{|l|}{$\mathrm{f}_{1}$} & \multicolumn{2}{|l|}{$\mathrm{f}_{2}$} & \multicolumn{2}{|l|}{$\mathrm{f}_{3}$} & $\mathrm{f}_{4}$ & \\
\hline Scenario 40 & \multicolumn{3}{|l|}{$f_{1}$} & $\mathrm{f}_{2}$ & & \multicolumn{2}{|l|}{$\mathrm{f}_{3}$} & \\
\hline Scenario 41 & \multicolumn{4}{|l|}{$\mathrm{f}_{1}$} & \multicolumn{2}{|l|}{$\mathrm{f}_{2}$} & $\mathrm{f}_{3}$ & \\
\hline Scenario 42 & \multicolumn{5}{|l|}{$f_{1}$} & \multicolumn{2}{|l|}{$\mathrm{f}_{2}$} & $\mathrm{f}_{3}$ \\
\hline Scenario 43 & \multicolumn{2}{|l|}{$f_{1}$} & \multicolumn{3}{|l|}{$\mathrm{f}_{2}$} & \multicolumn{2}{|l|}{$f_{3}$} & $\mathrm{f}_{4}$ \\
\hline Scenario 44 & \multicolumn{2}{|l|}{$f_{1}$} & \multicolumn{4}{|l|}{$\mathrm{f}_{2}$} & \multicolumn{2}{|l|}{$\mathrm{f}_{3}$} \\
\hline Scenario 45 & \multicolumn{2}{|l|}{$\mathrm{f}_{1}$} & \multicolumn{5}{|l|}{$\mathrm{f}_{2}$} & $\mathrm{f}_{3}$ \\
\hline Scenario 46 & \multicolumn{2}{|l|}{$f_{1}$} & \multicolumn{2}{|l|}{$f_{2}$} & \multicolumn{3}{|l|}{$f_{3}$} & $\mathrm{f}_{4}$ \\
\hline Scenario 47 & \multicolumn{2}{|l|}{$\mathrm{f}_{1}$} & $\mathrm{f}_{2}$ & & \multicolumn{4}{|l|}{$f_{3}$} \\
\hline Scenario 48 & \multicolumn{3}{|l|}{$\mathrm{f}_{1}$} & $f_{2}$ & & & \multicolumn{2}{|l|}{$f_{3}$} \\
\hline Scenario 49 & \multicolumn{4}{|c|}{$f_{1}$} & \multirow{2}{*}{\multicolumn{3}{|c|}{$\mathrm{f}_{2}$}} & $\mathrm{f}_{3}$ \\
\hline Scenario 50 & \multicolumn{3}{|l|}{$f_{f}$} & $\mathrm{f}_{2}$ & & & & $\mathrm{f}_{3}$ \\
\hline
\end{tabular}

Table 10. DBIM Using Dual-Frequency (DF-DBIM)

\begin{tabular}{|l|l|l|l|l|l|l|l|l|}
\hline Iterations & $\mathbf{1}$ & $\mathbf{2}$ & $\mathbf{3}$ & $\mathbf{4}$ & $\mathbf{5}$ & $\mathbf{6}$ & $\mathbf{7}$ & $\mathbf{8}$ \\
\hline Scenario 51 & $\mathrm{f}_{1}$ & $\mathrm{f}_{2}$ & \multicolumn{7}{|l|}{} \\
\hline Scenario 52 & $\mathrm{f}_{1}$ & $\mathrm{f}_{2}$ & $\mathrm{f}_{2}$ \\
\hline Scenario 53 & $\mathrm{f}_{1}$ & $\mathrm{f}_{1}$ & $\mathrm{f}_{2}$ & \\
\hline Scenario 54 & $\mathrm{f}_{1}$ & $\mathrm{f}_{1}$ & $\mathrm{f}_{2}$ & $\mathrm{f}_{2}$ \\
\hline Scenario 55 & $\mathrm{f}_{1}$ & & & $\mathrm{f}_{2}$ \\
\hline Scenario 56 & $\mathrm{f}^{2}$ &
\end{tabular}

Table 11. The normalized error of 57 scenarios from Table 2 to 11 after eight iterations

\begin{tabular}{|l|l|l|l|l|l|l|l|l|l|l|}
\hline Scenarios & $\mathbf{1}$ & $\mathbf{2}$ & $\mathbf{3}$ & $\mathbf{4}$ & $\mathbf{5}$ & $\mathbf{6}$ & $\mathbf{7}$ & $\mathbf{8}$ & $\mathbf{9}$ & $\mathbf{1 0}$ \\
\hline Error & 0.0728 & 0.0781 & 0.0882 & 0.1157 & 0.1536 & 0.0787 & 0.0882 & 0.0976 & 0.1248 & 0.1730 \\
\hline Scenarios & 11 & 12 & 13 & 14 & 15 & 16 & 17 & 18 & 19 & 20 \\
\hline Error & 0.0785 & 0.0902 & 0.0988 & 0.1215 & 0.1275 & 0.0821 & 0.0881 & 0.0979 & 0.1113 & $\mathbf{0 . 0 7 5 7}$ \\
\hline Scenarios & 21 & 22 & 23 & 24 & 25 & 26 & 27 & 28 & 29 & 30 \\
\hline Error & 0.0896 & 0.0885 & 0.0774 & 0.0825 & 0.0848 & 0.0897 & 0.1143 & 0.1528 & 0.0898 & 0.1009 \\
\hline Scenarios & 31 & 32 & 33 & 34 & 35 & 36 & 37 & 38 & 39 & 40 \\
\hline Error & 0.1495 & 0.0866 & 0.0947 & 0.1206 & 0.1136 & 0.0950 & 0.1235 & 0.1137 & 0.0923 & 0.1077 \\
\hline Scenarios & 41 & 42 & 43 & 44 & 45 & 46 & 47 & 48 & 49 & 50 \\
\hline Error & 0.0955 & 0.1477 & 0.1096 & 0.1090 & 0.1527 & 0.1096 & 0.0939 & 0.0981 & 0.1420 & 0.1500 \\
\hline Scenarios & 51 & 52 & 53 & 54 & 55 & 56 & 57 & $\#$ & $\#$ & $\#$ \\
\hline Error & 0.2037 & 0.1472 & 0.1339 & 0.1293 & 0.1352 & 0.1507 & 0.2030 & $\#$ & $\#$ & $\#$ \\
\hline
\end{tabular}




\section{CONCLUSION}

This paper successfully developed a multi-frequency DBIM image reconstruction model termed FTaOT that yields a promising result. The normalized error after each iteration has a notable improvement. Multi-frequency DBIM certainly takes more time than single-frequency DBIM, but the difference is insignificant. We performed 57 scenarios comparing the proposed method to multifrequency DBIM to prove our method's reliability, in which our approach gains a better result (in Tables $1-11)$. This work can be further developed by 3D reconstruction and experiment.

\section{FUNDING}

This work was supported by Vietnam's Ministry of Education and Training under Grant No. B2020SP2-03. 


\section{REFERENCES}

Feng, . (Ed.). (2019). Biomedical Information Technology. Academic Press.

Golub, G. H., Hansen, P. C., \& O’Leary, D. P. (1999). Tikhonov regularization and total least squares. SIAM Journal on Matrix Analysis and Applications, 21(1), 185-194. doi:10.1137/S0895479897326432

Haddadin, O. S., \& Ebbini, E. S. (1997). Acoustical Imaging: Multiple frequency distorted Born iterative method for tomographic imaging. Springer.

Haddadin, O. S., \& Ebbini, E. S. (1998). Imaging strongly scattering media using a multiple frequency distorted Born iterative method. IEEE Transactions on Ultrasonics, Ferroelectrics, and Frequency Control, 45(6), 1485-1496. doi:10.1109/58.738288 PMID:18249996

Jonathan, M., \& Oelze, M. L. (2013). Quantitative ultrasound in soft tissues. Springer.

Kino, G. S. (1987). Acoustic Waves: Devices, Imaging, and Analog Signal Processing. Prentice Hall.

Lavarello, R., \& Oelze, M. (2010). Density imaging using a multiple-frequency DBIM approach. IEEE Transactions on Ultrasonics, Ferroelectrics, and Frequency Control, 57(11), 2471-2479. doi:10.1109/ TUFFC.2010.1713 PMID:21041134

Ma, T., Yu, M., Chen, Z., Fei, C., Shung, K. K., \& Zhou, Q. (2015). Multi-frequency intravascular ultrasound (IVUS) imaging. IEEE Transactions on Ultrasonics, Ferroelectrics, and Frequency Control, 62(1), 97-107. doi:10.1109/TUFFC.2014.006679 PMID:25585394

Macovski, A. (1979). Ultrasonic imaging using arrays. Proceedings of the IEEE, 67(4), 484-495. doi:10.1109/ PROC.1979.11278

Montero, R. J. L., \& Janniel, R. (2009). New developments on quantitative imaging using ultrasonic waves $(\mathrm{PhD}$ Thesis). University of Illinois at Urbana-Champaign.

National Research Council. (2006). Visualizing Chemistry, The progress and promise of advanced chemical imaging. National Academies Press.

Sayed. (2018). Multi-frequency ultrasound imaging. International Journal of Allied Health Sciences, 2(2), 304-309.

Schueler, C. F., Lee, H., \& Wade, G. (1984). Fundamentals of digital ultrasonic processing. IEEE Transactions on Sonics and Ultrasonics, 31(4), 195-217. doi:10.1109/T-SU.1984.31501

Slaney, M., Kak, A. C., \& Larsen, L. E. (1984). Limitations of imaging with first-order diffraction tomography. IEEE Transactions on Microwave Theory and Techniques, 32(8), 860-874. doi:10.1109/TMTT.1984.1132783

Tijhuis, A. G., Belkebir, K., Litman, A. C., \& de Hon, B. P. (2001). Multiple-frequency distorted-wave Born approach to 2D inverse profiling. Inverse Problems, 17(6), 1635-7.

Tran, Q. H., Tran, D. T., Huynh, H. T., Ton-That, L., \& Nguyen, L. T. (2016). Influence of dual-frequency combination on the quality improvement of ultrasound tomography. Simulation, 92(3), 267-276. doi: $10.1177 / 0037549716630605$

Varray, F., Cachard, C., Kybic, J., Novell, A., Bouakaz, A., \& Basset, O. (2012). A multi-frequency approach to increase the native resolution of ultrasound images. Proceedings of the 20th European Signal Processing Conference (EUSIPCO), 2733-2737.

Yoshizumi, N., Saito, S., Koyama, D., Nakamura, K., Ohya, A., \& Akiyama, I. (2009). Multiple-frequency ultrasonic imaging by transmitting pulsed waves of two frequencies. Journal of Medical Ultrasonics, 36(2), 53-60. doi:10.1007/s10396-009-0213-7 PMID:27277084

Zhu, Q., \& Steinberg, B. D. (1993, November). Wavefront amplitude distortion and image sidelobe levels: Part I - Theory and computer simulations. IEEE Transactions on Ultrasonics, Ferroelectrics, and Frequency Control, 40(6), 747-753. doi:10.1109/58.248219 PMID:18263242 
Tran Quang-Huy was born in Hanam, Vietnam, in 1985. He received the B.Sc. degree in physics from the Hanoi Pedagogical University No2 (HPU2), in 2009, and the M.Sc degree in Electrical engineering from the VNU-UET, in 2013. He is currently a lecturer in the Faculty of Physics, HPU2, and also a PhD student of the VNU-UET. His research interest is biomedical signal processing such as Ultrasound tomography (UT), Magnetic resonance imaging (MRI), Electron paramagnetic resonance (EPR).

Vijender Kumar Solanki, Ph.D., is an Associate Professor in Computer Science \& Engineering, CMR Institute of Technology (Autonomous), Hyderabad, TS, India. He has more than 10 years of academic experience in network security, IoT, Big Data, Smart City and IT. Prior to his current role, he was associated with Apeejay Institute of Technology, Greater Noida, UP, KSRCE (Autonomous) Institution, Tamilnadu, India \& Institute of Technology \& Science, Ghaziabad, UP, India. He has authored or co-authored more than 25 research articles that are published in journals, books and conference proceedings. He has edited or co-edited 4 books in the area of Information Technology.. He received Ph.D in Computer Science and Engineering from Anna University, Chennai, India in 2017 He is Editor in International Journal of Machine Learning and Networked Collaborative Engineering (IJMLNCE) ISSN 2581-3242, Associate Editor in International Journal of Information Retrieval Research (IJIRR), IGI-GLOBAL, USA, ISSN: 2155-6377 | E-ISSN: 2155-6385. He is guest editor with IGI-Global, USA, InderScience \& Many more publishers.

Duc-Tan Tran is an Associate professor and Vice Dean of Faculty of Electrical and Electronic Engineering, Phenikaa University. He has published over 150 research papers. His publications received the "Best Paper Award" at the 9th International Conference on Multimedia and Ubiquitous Engineering (MUE-15), and International Conference on Green and Human Information Technology (ICGHIT-2015). He was the recipient of the award for the excellent young researcher from Vietnam National University in 2008, Hanoi and the third prize in the contest "Vietnamese Talents" in 2008. His main research interests include the representation, processing, analysis, and communication of information embedded in signals and datasets. He serves as a TP Co-chair, technical committee program member, track chair, session chair and reviewer of many international conferences and journals. 